\title{
Ajustes razonables en la universidad. Creencias, prác- ticas y dificultades para llevarlos a cabo desde la voz de docentes inclusivos
}

\section{María Nieves Sánchez Díaz'}

Resumen: Las universidades españolas tienen el gran reto de garantizar los principios de igualdad de oportunidades, no discriminación y accesibilidad de todos los estudiantes. Este estudio se centra en conocer las creencias y acciones del profesorado universitario para responder a las necesidades del alumnado con discapacidad, así como en detectar las necesidades del profesorado para realizar modificaciones en sus asignaturas. Participaron 42 docentes seleccionados como inclusivos por un grupo de estudiantes con discapacidad. Todos ellos pertenecían a 6 universidades públicas españolas e impartían docencia en la Facultad de Ciencias de la Educación. Se llevó a cabo un diseño cualitativo basado en el método biográfico. Los resultados evidencian que el profesorado presenta una actitud generalmente positiva hacia la realización de ajustes razonables en el aula y los ajustes razonables más aplicados son aquellos referidos al acceso al currículum. Se identifican algunas necesidades para seguir avanzando en materia de inclusión en Educación Superior.

Palabras clave: Concepciones; Prácticas docentes;Ajustes Razonables; Pedagogía Inclusiva; Educación Superior.

Fecha de recepción: I de febrero de 2021 .

Fecha de admisión definitiva: 22 de marzo de 202I.

' Universidad de Sevilla. Departamento de Psicología Evolutiva y de la Educación. 
Reasonable adjustments at the university. Beliefs, practices and difficulties in applying them from the voice of inclusive educators

Abstract: Spanish universities have the great challenge of guaranteeing the principles of equal opportunities, non-discrimination and accessibility for all students. This study focuses on understanding the beliefs and actions of university faculty in order to respond to the needs of students with disabilities, as well as to detect the needs of faculty to make modifications in their subjects. 42 faculty members deemed inclusive by a group of students with disabilities participated in the study. All of them belonged to 6 Spanish public universities and taught at the Faculty of Educational Sciences. A qualitative design was carried out based on the biographical method. The results show that faculty generally have a positive attitude when it comes to accommodating students in reasonable ways in the classroom, and that the most frequently needed reasonable adjustments are related to access to the curriculum. Some needs have been identified in order to make further progress in the field of inclusion in Higher Education.

Keyswords: Conceptions; Faculty Practices; Reasonable Adjustments; Inclusive Pedagogy; Higher Education.
Ajustements raisonnables à l'université. Croyances, pratiques et difficultés à les mettre en œuvre de la voix des enseignants inclusifs

Résumé: Les universités espagnoles ont le grand défi de garantir les principes d'égalité des chances, de non-discrimination et d'accessibilité pour tous les étudiants. La présente étude se concentre sur la compréhension des croyances etactions du corps des enseignants de l'université pour répondre aux besoins des personnes handicapées, et la détection des besoins des enseignants pour apporter des modifications dans leurs matières. 48 enseignants jugés inclusifs par un groupe d'étudiants handicapés ont été sélectionnés pour participer. Ils appartenaient tous à 6 universités espagnoles et enseignaient à la faculté des sciences de l'éducation. Une conception qualitative a été utilisée, basée sur la méthode biographique. Les résultats montrent que le corps d'enseignants a généralement une attitude positive à l'égard d'un accommodement raisonnable de la salle de classe et que les aménagements raisonnables les plus couramment appliqués sont ceux liés à l'accès au programme d'études.

Mots clé: Conceptions; pratiques de la faculté; ajustements raisonnable; pédagogie inclusive; éducation supérieure.

\section{Introducción}

La presencia de estudiantes con discapacidad en las aulas universitarias españolas ha aumentado significativamente en las últimas décadas. Según los datos que ofrece la Fundación Universia, el número de estudiantes con discapacidad que accede y cursa estudios universitarios ha aumentado de 12.755 en 2013 a 21.435 en 2018 (Fundación Universia, 2018), suponiendo un incremento del $68,05 \%$ en 5 años. Esta realidad requiere que las universidades se conviertan 
en contextos accesibles e inclusivos, en los que se respondan a las necesidades de todo el alumnado.

La Pedagogía Inclusiva se presenta como una aproximación pedagógica que favorece el camino hacia una universidad accesible y pensada para todos y todas (Moriña, 2020). Esta aproximación teórico-práctica, parte de cuatro dimensiones de análisis: creencias, conocimientos, diseños y acciones (Florian, 2014; Gale y Mills, 2013; Rouse, 2009), que permiten conocer cómo, por qué y para qué desarrolla el profesorado prácticas inclusivas. En este trabajo, se pretenden conocer las creencias y las acciones del profesorado universitario inclusivo, partiendo de la idea de que una creencia es una acción simbólica que adquiere significación cuando se pone en marcha la propia acción (Defez, 2005).

En España, el acceso para el alumnado con discapacidad a la universidad se encuentra amparado legalmente. La Ley Orgánica de Universidades (LOU 4/2007, de 12 de abril), establece que deben garantizarse los principios de igualdad de oportunidades y no discriminación por razón de discapacidad, y determina que los edificios e instalaciones universitarias deben ser accesibles. Del mismo modo, el Real Decreto Legislativo por el que se aprueba el Texto Refundido de la Ley General de derechos de las personas con discapacidad y de su inclusión social (RDL 1/2013, de 29 de noviembre), regula el derecho de igualdad de oportunidades y no discriminación de estos estudiantes, establece que los centros universitarios deben contar con los apoyos necesarios para la inclusión del alumnado con discapacidad y menciona la necesidad de incluir en las distintas titulaciones universitarias planes de formación en Diseño Universal o Diseño para todas las personas.

Estas normativas incluyen los principios de igualdad de oportunidades, no discriminación y accesibilidad universal para el alumnado con discapacidad. Sin embargo, la realidad es muy distinta en la práctica (Moriña et al., 2020). La universidad continúa siendo un ambiente hostil para el alumnado con discapacidad, en tanto en cuanto siguen existiendo barreras -físicas, de acceso al currículum, actitudinales y políticas- que impiden su participación en igualdad de condiciones, y, por ende, su inclusión real (Sánchez-Díaz y Morgado, en prensa, 2021 ; Langørgen y Magnus, 2018). Entre estas barreras, la actitud negativa por parte de algunos docentes ha sido identificada como una de las más importantes (Gibson, 2015; Moriña et al., 2015), junto con la existencia de planes de estudio rígidos e inflexibles.

En la universidad, las tasas de ingreso, permanencia y éxito del alumnado con discapacidad son más bajas que las del resto de estudiantes (Gairín et al., 2013; Lightfoot et al., 2018; Seale et al., 2020). Ante esta realidad, una medida para 
facilitar la inclusión de estos estudiantes en el aula consiste en realizar ajustes razonables en las materias que así lo requieran. Se entiende por ajustes razonables aquellas modificaciones y adaptaciones necesarias a nivel físico, social y actitudinal que aborden las necesidades específicas de las personas con discapacidad, para facilitar su accesibilidad y participación en igualdad de condiciones (RDL 1/2013, de 29 de noviembre). En un intento por hacer una clasificación de aquellos ajustes que pueden realizarse en el contexto universitario, estos se clasifican, siguiendo a Alcantud et al. (2000), en:

- Adaptaciones de acceso. Incluyen ajustes de acceso físico, que implican la eliminación de barreras arquitectónicas, la adecuación de iluminación y sonido en el aula, la provisión de material adaptado o de personal de apoyo especializado; y adaptaciones de acceso a la comunicación, como, por ejemplo, apoyo tecnológico, sistemas de comunicación alternativos, adaptación de textos, imágenes o señalética.

- Adaptaciones individualizadas. Incluyen la modificación de los elementos propios del currículum. Pueden ser no significativas, que implican modificaciones en las actividades, los tiempos, la metodología o el tipo de evaluación; o significativas, que afectan a los contenidos, objetivos o criterios de evaluación, manteniendo las competencias generales del título. A este respecto, la realización de adaptaciones curriculares significativas no está permitida en universidades públicas españolas, a no ser que las propias universidades dispongan de programas especiales que permitan la realización de las mismas.

Ahora bien, aunque los ajustes razonables son una buena medida para garantizar los principios de igualdad de oportunidades y accesibilidad del alumnado con discapacidad en la universidad, diversos autores coinciden en que no serían necesarios si se diseñasen los proyectos docentes teniendo en cuenta desde el inicio los principios del Diseño Universal de Aprendizaje (DUA), ya que este enfoque permite diseñar la enseñanza de manera que ningún estudiante se quede fuera de ella, permitiendo y garantizando la participación de todo el alumnado (Bunbury, 2020; Fichten et al., 2016; Schreiber, 2017).

Así pues, el DUA aparece como un enfoque que facilita la inclusión educativa en las aulas universitarias, huyendo de planes de estudios rígidos que dificultan el acceso y la participación de algunos estudiantes (CAST, 2018; Meyer et al., 2014) y evitando el tener que hacer ajustes sobre una planificación ya establecida. Este marco de aplicación se sustenta en tres principios fundamentales: 
- Ofrecer múltiples formas de representación: Proporcionar opciones para la percepción, la comprensión, el lenguaje, los símbolos y las expresiones matemáticas.

- Ofrecer múltiples formas de expresión y acción: Proporcionar opciones para la interacción física, para la expresión y la comunicación y para facilitar las funciones ejecutivas.

- Ofrecer múltiples formas de motivación: Proporcionar diversas opciones para captar el interés, para la autorregulación y para mantener el esfuerzo y la persistencia.

A pesar de ser una línea de investigación reciente, diversos estudios se han centrado en conocer cómo se desarrolla el DUA en el marco de la Educación Superior (Boothe et al., 2018; Fleet y Kondrashov, 2019), así como en diseñar, implementar y evaluar programas de formación específicos en esta temática para el profesorado universitario (Hromalik et al., 2020; Hsiao et al., 2019; Moriña y Carballo, 2018; Rodesiler y McGuire, 2015). Estos estudios cobran especial relevancia, ya que, se ha podido comprobar que, en ocasiones, el profesorado muestra una actitud negativa, a veces por desconocimiento y falta de formación, para realizar ajustes razonables en sus materias conducentes a abordar las necesidades del alumnado con discapacidad (Banks, 2019; Hong, 2015; Lightfoot et al., 2018; Toutain, 2019).

Y es que, la formación del profesorado es un aspecto clave si se pretende avanzar hacia la inclusión del alumnado con discapacidad (Debrand y Salzberg, 2005; Lombardi et al., 2013), ya que la falta de capacitación del profesorado en cuestiones relacionadas con la atención a la diversidad puede suponer una barrera para estos estudiantes. Son muchos los estudios que evidencian esta necesidad de formación por parte del profesorado para atender correctamente al alumnado con discapacidad (Hsiao et al., 2019), y autores como Moriña y Carballo (2018) han encontrado en sus investigaciones que es el propio profesorado quien verbaliza su propia necesidad de formación específica para poder ajustar su actuación docente a las características de este alumnado, realizando así los ajustes razonables que sean necesarios.

A pesar de ello, existen estudios que han encontrado que el profesorado presenta actitudes generalmente positivas en la aplicación de estas modificaciones (Abdella, 2018; Elbeheri et al., 2018; Greenberger, 2016), las cuales reportan beneficios muy significativos para el alumnado, como el aumento del rendimiento, de la participación, de la expresión e incluso de las calificaciones de los estudiantes (Dalton, 2017; Schrever y Sachs, 2014). 
Para finalizar, cabe mencionar la escasez de investigaciones centradas en conocer los ajustes razonables que se realizan en las aulas universitarias (Melero et al., 2019; Sandoval et al., 2020), por lo que este trabajo contribuye a cubrir una laguna detectada en la literatura científica.

Este artículo tiene como objetivo analizar las creencias del profesorado sobre la necesidad de realizar ajustes en sus materias para mejorar el aprendizaje de sus estudiantes; conocer qué ajustes han llevado a la práctica en sus asignaturas cuando han tenido estudiantes con discapacidad; e identificar las dificultades o necesidades del profesorado para realizar estas modificaciones o ajustes. Tres preguntas de investigación guiaron el análisis: 1) ¿ Qué opinión tiene el profesorado participante sobre la necesidad de realizar ajustes razonables en sus asignaturas?, 2) ¿Qué tipo de modificaciones ha realizado el profesorado universitario inclusivo para dar respuesta a las necesidades del alumnado con discapacidad que cursa sus asignaturas? y 3) ¿Qué dificultades o necesidades encuentra el profesorado para realizar modificaciones en sus asignaturas?

\section{Método}

El presente estudio forma parte de una investigación más amplia "Pedagogía Inclusiva en la Universidad: Narrativas del Profesorado (Ref. EDU2016-76587-R)" financiada por el Ministerio de Ciencia e Innovación del Gobierno de España y la Agencia Estatal de Investigación y cofinanciada por fondos FEDER, centrada en conocer las creencias, los conocimientos, los diseños y las acciones de profesorado universitario que desarrolla prácticas inclusivas. En este artículo se analizan las creencias y las acciones de estos docentes.

La investigación se enmarca en el paradigma cualitativo. Concretamente en el diseño de investigación biográfico-narrativo, centrado, en este caso, en la importancia de poner en primer plano la experiencia del profesorado universitario inclusivo. En este estudio participaron un total de 42 profesores de 6 universidades públicas españolas. En cuanto a la selección de los participantes, estos fueron seleccionados exclusivamente por estudiantes universitarios con discapacidad. Para ello, se pidió colaboración a los Servicios de Apoyo a la Discapacidad de las universidades participantes. El personal técnico de estos servicios contactó con los estudiantes con discapacidad y les proporcionó información sobre el proyecto para que nombraran de manera voluntaria a aquellos docentes que desarrollaban prácticas inclusivas. Para este fin, y tras la lectura de diversos estudios centrados en conocer las ca- 
racterísticas de docentes inclusivos (por ejemplo, el estudio de Kioko y Makoelle (2014)), el equipo de investigación se reunió para fijar una serie de criterios que debían tener estos docentes y elaborar una lista que se proporcionó posteriormente al alumnado con discapacidad. Estos criterios de selección fueron los siguientes: Cree en las posibilidades de todos los estudiantes; facilita los procesos de aprendizaje; su enseñanza es activa, utilizando diferentes estrategias metodológicas de enseñanza; muestra preocupación por el aprendizaje de sus estudiantes; muestra flexibilidad, con voluntad de ayudar; motiva a los estudiantes; mantiene relaciones cercanas y favorece las interacciones entre estudiantes; te hace sentir que eres importante, que eres uno más en el aula; permite que los estudiantes participen en la clase y construir el conocimiento juntos; la comunicación que mantiene contigo y con tus compañeros es horizontal.

Una vez que el alumnado proporcionó los nombres de aquellos docentes que desarrollaban prácticas inclusivas en sus aulas, se contactó con ellos y ellas mediante correo electrónico. Se contactó, en un principio, con un total de 66 docentes, de los que finalmente accedieron a participar 42 . A estos 42 docentes se les envió una carta informativa del estudio y un documento de Consentimiento Informado que debían firmar para participar. La información personal de los participantes fue codificada y custodiada para cumplir con los criterios de anonimato y confidencialidad.

En cuanto al perfil de los participantes, del total de docentes ( $N=42)$, el $40,5 \%$ fueron hombres $(\mathrm{N}=17)$ y el $59,5 \%$ mujeres $(\mathrm{N}=25)$. En relación a la edad, esta oscilaba entre los 33 y los 59 años, siendo la media igual a 41,2 años. Respecto a la práctica docente, los participantes contaban entre 7 y 32 años de experiencia, siendo la media de 15,8 años de experiencia docente. Todo el profesorado participante tenía experiencia previa con estudiantes con discapacidad.

\section{I. El contexto universitario español}

Los docentes que participaron en este estudio pertenecían a seis universidades públicas de España. Siguiendo la distribución realizada desde el Espacio Europeo de Educación Superior, las titulaciones universitarias oficiales se dividen en Estudios de Grado, de 4 años de duración, y Estudios de Posgrado, donde se enmarcan Másteres Universitarios y Estudios de Doctorado.

En España, el Real Decreto por el que se aprueba el Estatuto del Estudiante Universitario (RD 1791/2010, de 30 de diciembre), dispone que, dentro de la normativa establecida por las distintas universidades, se deben poner a disposi- 
ción del alumnado con discapacidad los medios materiales, humanos y técnicos necesarios para garantizar la igualdad de oportunidades y la integración plena en la comunidad universitaria. De este modo, todas las universidades públicas disponen de Oficinas de Apoyo para el alumnado con discapacidad, las cuales se encargan de que estos estudiantes dispongan de los recursos necesarios para el desarrollo de su proceso de aprendizaje y asesoran al personal académico sobre los diferentes ajustes razonables que han de realizar, en el caso que sea necesario.

\subsection{Instrumento de recogida de datos y procedimiento}

La investigación se realizó a partir de dos entrevistas individuales semiestructuradas diseñadas para el estudio donde se exploraron tres cuestiones: 1) Opinión sobre la necesidad de realizar ajustes razonables a estudiantes universitarios; 2) Ajustes razonables que el profesorado ha aplicado a sus materias para hacerlas accesibles al alumnado con discapacidad y 3) Necesidades o dificultades encontradas por el profesorado para ajustar su enseñanza a las necesidades del alumnado con discapacidad.

Las entrevistas fueron realizadas por los miembros del equipo de investigación formados previamente para dicha tarea. La mayor parte de estas fueron realizadas cara a cara $(n=34)$. No obstante, por dificultades diversas, a 6 profesores se les realizó la entrevista vía Skype y a 2 vía telefónica. Las entrevistas tuvieron una duración media de 90 minutos. Todas las entrevistas fueron grabadas en audio y los docentes mostraron por escrito su consentimiento a la grabación y el uso de los datos para la investigación. Además, este estudio cumplió con los requisitos éticos aprobados por el Ministerio de Economía y Competitividad de España.

\subsection{Análisis de datos}

La información fue literalmente transcrita y analizada cualitativamente mediante un sistema de categorías y códigos inductivo que posibilitó organizar y dar coherencia a la información recogida (Miles y Huberman, 1994). Las categorías y códigos utilizados para el análisis se muestran en la siguiente tabla (Tabla 1). 


\section{TABLA I. Categorías y códigos de análisis}

\begin{tabular}{|l|l|l|}
\hline \multicolumn{1}{|c|}{$\begin{array}{c}\text { Categoría } \\
\text { 1. Creencias sobre } \\
\text { adaptaciones } \\
\text { curriculares. }\end{array}$} & $\begin{array}{l}\text { Subcategoría } \\
\text { 1.1. Opinión } \\
\text { adaptaciones. }\end{array}$ & $\begin{array}{l}\text { Códigos } \\
\text { COA1. Garantizar la inclusión de todo el } \\
\text { alumnado. } \\
\text { COA2. Garantizar la inclusión de todo el } \\
\text { alumnado sin disminuir la exigencia. } \\
\text { COA3. Nonecesidad de realizar adaptaciones. }\end{array}$ \\
\hline $\begin{array}{l}\text { 2. Adaptaciones } \\
\text { de la materia. }\end{array}$ & 2.1. Adaptaciones. & $\begin{array}{l}\text { CA1. Diseño de asignaturas siguiendo prin- } \\
\text { cipios del DUA. } \\
\text { CA2. Realización de adaptaciones de acceso. } \\
\text { CA3. Realización de adaptaciones individuales. }\end{array}$ \\
\hline & $\begin{array}{l}\text { 2.2. Dificultades } \\
\text { adaptaciones. }\end{array}$ & $\begin{array}{l}\text { CDA1. Necesidad de formación para realizar } \\
\text { adaptaciones. } \\
\text { CDA2. Necesidad de información sobre las }\end{array}$ \\
\hline
\end{tabular}

Elaboración propia.

\section{Resultados}

Los resultados de este trabajo permiten conocer las creencias que tiene el profesorado inclusivo sobre la realización de ajustes razonables, así como las adaptaciones que han aplicado en sus materias para abordar las necesidades de estudiantes universitarios con discapacidad, y posibilitan detectar algunas necesidades o dificultades que tiene el profesorado cuando realiza modificaciones en sus asignaturas.

\section{I. Creencias del profesorado universitario sobre la necesidad de realizar ajustes razonables en el aula}

El primer objetivo de este trabajo era analizar las creencias del profesorado sobre la necesidad de realizar ajustes razonables en sus materias.

En general, el profesorado participante presentaba una actitud positiva a realizar ajustes razonables en el aula. Entre los motivos con los que argumentaban la ne- 
cesidad de realizar estos ajustes se encontraba, fundamentalmente, la necesidad de atender a la diversidad de todo el alumnado, independientemente de tener, o no, una discapacidad, garantizando así el principio de igualdad de oportunidades y no discriminación.

"Claro, es totalmente necesario, sí, sí, ya digo, para alumnos con discapacidad como si a lo mejor hay un alumno que, por lo que sea, pues no puede venir a clases porque tiene que trabajar, también hay que hacerle algún otro tipo de adaptación, o sea, que es totalmente fundamental" (Participante 6).

"Estoy firmemente a favor de las adaptaciones curriculares porque es que no creo que, porque un contenido no pueda ser visto por el alumno con discapacidad de la misma forma que otro, que ese alumno con discapacidad no tenga las mismas oportunidades de aprobar el examen, o lo que sea. Eso en todas las asignaturas. Bueno, podrá hacer y aprender otras cosas, y podrá ejercer" (Participante 42).

No obstante, muchos de estos docentes incluían en sus argumentos una condición que debía cumplirse a la hora de realizar estos ajustes, y es que se garantizase que los ajustes no implicasen diferencias entre el alumnado que los requería y el resto de estudiantes, ya que esto perjudicaría al alumnado al que se le aplicasen estos ajustes, que podría autopercibirse diferente a los demás.

"En la universidad, lo que pasa que una adaptación a nivel de contenidos no está contemplada. Entonces, es una adaptación a nivel estructural, de organización o de una pauta que necesiten. Pero, cuando hicimos la reunión estaba claro, los contenidos tenían que superarlos como cualquier otro" (Participante 20).

"Yo soy más partidario de adaptar aspectos de llegar al currículum común para todos, pero partiendo de que es importante que todo el mundo aprenda lo mismo, porque los estudiantes entienden enseguida que son diferentes, entonces, si tú trabajas de una forma diferente, la autoestima del propio alumno queda mal, pero la percepción que tiene el resto del alumnado de esta persona, también es bastante negativa. Lo que es eliminar o hacer algo diferente del resto, no soy nada partidario" (Participante 21).

Solo un docente manifestaba que no era necesario realizar ajustes razonables en la universidad, argumentando, por un lado, que el excesivo número de estudiantes que existen en las aulas universitarias dificultaba la atención individualizada, y por otro, que existía una baja probabilidad de tener en el aula estudiantes con discapacidad.

"Es que, yo no lo veo. No lo veo, no por nada, sino porque hay que ser realistas y porque tenemos una media de 70 alumnos por clase, y es raro que en la universidad te aparezcan casos de discapacidad" (Participante 8). 


\subsection{Prácticas inclusivas en el aula. Ajustes razonables desarrollados por el profesorado inclusivo}

El segundo objetivo de este estudio era conocer las modificaciones que el profesorado inclusivo ha llevado a cabo en sus asignaturas cuando han tenido estudiantes con discapacidad.

Del total de profesores, solo cuatro docentes han manifestado tener su asignatura diseñada desde el primer momento teniendo en cuenta los principios del Diseño Universal de Aprendizaje, argumentando que, a pesar de ser una ardua tarea, el modelo permitía que todo el alumnado se sintiera incluido en el aula. Estos docentes contemplaban la diversidad que podía existir en un aula universitaria desde el primer momento en que diseñaban y recopilaban el contenido que iban a proporcionar al alumnado. A pesar de ello, durante el desarrollo de sus asignaturas, reconocían estar pendientes de las necesidades que pudieran surgir.

"Intento trabajar, eso sí, con distintos materiales y de distintas maneras para atender a la mayor parte de situaciones diversas que tengo dentro de mi aula... Intento que los vídeos que pongo en clase estén subtitulados, trabajo con otro tipo de recursos audiovisuales y si tengo alguna necesidad le pongo también subtítulos y audio en voz, lo pongo con letras grandes ... Es decir, intento que el material esté adaptado a las múltiples realidades que pueda haber dentro de mi aula" (Participante 12).

"En nuestro caso no ha habido necesidad de adaptación. Nosotros hemos hecho un ajuste, hemos ajustado nuestra asignatura a todos y todas nuestras alumnas. Entonces, si es cierto que hemos estado supervisando el terreno" (Participante 16).

Respecto a las adaptaciones de acceso, más de la mitad del profesorado reconoce haber realizado estas adaptaciones en sus materias, ya fuese a nivel físico, como, por ejemplo, buscando aulas accesibles o permitiendo al alumnado que lo necesitase, sentarse en las primeras filas del aula; o de acceso a la comunicación, adaptando textos o enviando el material al alumnado por adelantado para que el propio estudiante pudiese adaptarlo a sus necesidades (cambiando el tamaño y el tipo de letra, imprimiéndolo en papel si es necesario, etc.).

"Por ejemplo, hay aulas que tienen las mesas o los pupitres fijos con sillas que se bajan, ¿no? Si no hay una mesa aparte suelta, una persona que viene en silla de ruedas no podría sentarse. Cuando tengo un aula donde hay una persona que no puede, o se busca otra mesa para que se pueda poner, o se solicita un cambio de aula porque hay aulas que sí están preparadas. El aula que te toca, te ha tocado, pero se reajusta, se cambia de lugar, se solicita otra aula..." (Participante 35).

"Pues, con los estudiantes que tenían discapacidad visual, he tenido que cambiar el formato de las presentaciones de Power Point, se las he dado con antelación, los he puesto en 
primera fila en clase, si han necesitado subir hasta donde está la pantalla para ver más cerca, pues les he dado libertad para hacerlo. En las clases prácticas, por ejemplo, de natación, al quitarse las gafas, ya sí que no veían nada, entonces tenían recursos básicos, como, por ejemplo, poner un cono fluorescente al final de la piscina para que supieran cuantos metros le quedaban para el final y que no se golpearan..." (Participante 10).

En cuanto a las adaptaciones individualizadas, algo más de un tercio del profesorado ha realizado modificaciones al diseño de su proyecto docente, incluyendo ajustes en los tiempos, actividades, metodología o tipo de evaluación. Estas adaptaciones, como puede apreciarse, no afectaban a los elementos básicos del currículum, por tanto, fueron adaptaciones individualizadas no significativas.

"Pues, no ya tanto en los contenidos, porque el tema tiene unas características y todos lo pueden desarrollar, pero sí a nivel de adaptación de esos contenidos $y$, fundamentalmente, a nivel metodológico y a nivel de evaluación. En esos casos debe haber unas adaptaciones curriculares no significativas" (Participante 9).

"Una alumna con discapacidad visual, me pedía que le hiciera los exámenes con una letra más grande, y su quince o veinte por ciento más de tiempo en el examen. Y, al final, lo que hacía era convocarla a ella en mi despacho porque allí en Magisterio, los turnos de exámenes, es que se acaba el examen y se acaba, y viene el siguiente, entonces, no dispones de más tiempo. Pues yo la convocaba a ella y le hacía el mismo examen que a los demás, el mismo tipo, pero con su letra grande y con el tiempo extra que necesitase" (Participante 41).

\subsection{Dificultades manifiestas por parte del profesorado para realizar ajustes razonables}

Respecto al tercer objetivo de este trabajo, muchos docentes han verbalizado necesidades y dificultades de diversa índole para realizar modificaciones en sus asignaturas, a pesar de considerar que la realización de dichas modificaciones o ajustes eran necesarias. Entre ellas, el profesorado verbalizaba la falta de formación que poseía para realizar estas modificaciones, lo que hacía que esta tarea les resultase dificultosa.

"Hombre, me parecen necesarias, el problema es si estamos de verdad formados para poder hacerlas. Hay un problema en la universidad de desinformación, o sea, ¿̇qué haces, una adaptación para todos los tipos de discapacidad que te puedes encontrar?, ¿lo haces a posteriori, cuando has visto al alumno o a la alumna, y ya estás metido en la tarea de ochenta alumnos por clase?, es que, me parece complicado, pero creo que es necesario" (Participante 3).

"Mi opinión es que es fundamental hacer adaptaciones. Ahora, otro problema grande es que pienso que, en la universidad, los profesores no estamos formados para hacer adaptaciones curriculares. Creo que el profesor debe contar con un servicio de apoyo, 
de asesoramiento, que ayude a hacer esas modificaciones, porque si no, el profesor nunca va a ser capaz de dar la talla en ese sentido" (Participante 11).

Otra de las dificultades manifestadas por el profesorado estaba relacionada con la información que recibían sobre el alumnado con discapacidad que asistía a sus aulas. A este respecto, el profesorado argumentaba que, a veces, no llegaba la información sobre las necesidades de los estudiantes con discapacidad, y que, en otras ocasiones, la información proporcionada por parte de los Servicios de Atención a la Discapacidad llegaba durante el desarrollo de la asignatura, impidiendo así que pudiesen poner en marcha las acciones necesarias para incluir a estos estudiantes.

"A veces me llega información del SACU, de algunos. De otros, pues me lo imagino, si no puede mover las manos, pues tendré que ayudarles a enfocar, ¿̇no?, si no pueden ver bien, pues lo mismo, ¿̇no?, otras veces me las he ingeniado yo" (Participante 1).

"Lo primero de todo, que los docentes sepan de su presencia, de su existencia, porque yo creo que es un problema importante que los docentes no sepan que tienen alumnos con discapacidad, y que los alumnos con discapacidad, por el motivo que sea, no se lo cuenten a sus profesores. $Y$, entonces, si no sabemos de su existencia, pues no se puede hacer nada" (Participante 41).

\section{Discusión y principales conclusiones}

Los resultados obtenidos posibilitan extraer conclusiones muy interesantes con respecto a los objetivos planteados en este trabajo y responder a las preguntas de investigación propuestas al inicio de este trabajo. Han permitido conocer las creencias del profesorado acerca de la necesidad de realizar ajustes razonables en sus materias, así como aquellas acciones que ponen en práctica en el aula para incluir al alumnado con discapacidad. Además, han ayudado a detectar algunas necesidades que tiene el profesorado para hacer modificaciones en sus asignaturas, aspecto fundamental si se quiere avanzar hacia universidades más inclusivas.

\section{I. ¿Qué opinión tiene el profesorado participante sobre la necesidad de realizar ajustes razonables en sus asignaturas?}

Respecto al primer objetivo de este trabajo, que hace referencia a la opinión del profesorado sobre la realización de ajustes razonables en sus asignaturas, los resultados muestran actitudes muy positivas hacia esta cuestión, a diferencia de los resultados encontrados por Banks (2019), Lightfoot et al. (2018) o Toutain (2019). 
El profesorado se muestra dispuesto a realizar las modificaciones necesarias, coincidiendo este dato con los hallados en trabajos previos (por ej., Abdella, 2018; Greenberger, 2016). Estos resultados pueden indicar una mayor sensibilidad de los participantes de este estudio ante la diversidad existente en las universidades españolas, frente a otros docentes de otros países tales como Estados Unidos o algunas regiones de Canadá (Banks, 2019; Lightfoot et al., 2018; Toutain, 2019). Además, algunas respuestas del profesorado aluden al principio de igualdad de oportunidades, principio inherente a la atención al alumnado con discapacidad, como se plantea tanto desde la Ley Orgánica de Universidades, (LOU, 4/2007, de 12 de abril) como desde el Real Decreto Legislativo por el que se aprueba el Texto Refundido de la Ley General de derechos de las personas con discapacidad y de su inclusión social (RDL 1/2013, de 29 de noviembre).Por su parte, y a pesar de la disposición positiva del profesorado hacia la realización de ajustes razonables que garanticen la inclusión de estudiantes con discapacidad, muchos docentes han puntualizado la necesidad de hacer estos ajustes teniendo en cuenta que no supongan en ningún momento una menor consideración al aprendizaje del alumnado, ni una disminución del nivel de exigencia, lo cual evidencia el compromiso del profesorado con la institución universitaria, considerada como la entidad educativa con mayor nivel de excelencia. Esta aportación es muy significativa, puesto que, sin conocerlo, el profesorado realiza una definición muy acertada de lo que supone realizar un ajuste razonable, en la línea de la definición que se refleja en la Guía de adaptaciones en la universidad, elaborada por la Red de Servicios de Apoyo a Personas con Discapacidad en la Universidad (SAPDU, 2017).

\section{2. ¿Qué tipo de modificaciones ha realizado el profesorado universitario inclusivo para dar respuesta a las necesidades del alumnado con discapacidad que cursa sus asignaturas?}

En lo que respecta a las acciones realizadas por el profesorado para incluir al alumnado con discapacidad en las aulas universitarias -el segundo objetivo de este trabajo- los resultados revelan que el profesorado realiza, fundamentalmente, adaptaciones de acceso, sin tener que realizar ajustes individuales sobre los elementos no prescriptivos o básicos del currículum. Dentro de estas adaptaciones de acceso, las adaptaciones de acceso a la comunicación son las más utilizadas, las cuales facilitan al alumnado la lectura y el manejo de textos, documentos y del contenido básico de las asignaturas. Estos resultados coinciden con los hallazgos de otros estudios centrados en estudiar los tipos de ajustes razonables que recibe el alumnado con discapacidad en la universidad, entre los cuales, destaca el trabajo de Mbuvha (2019). Con ello, desde este trabajo se pretende hacer un lla- 
mamiento a la calma hacia aquel profesorado inexperto o que se muestra reticente a realizar modificaciones en sus asignaturas, por cuestiones de cualquier índole, puesto que estos ajustes, en su mayoría, no requieren un gran esfuerzo por parte del profesorado y consiguen facilitar la trayectoria académica de este alumnado.

Por otro lado, y en la línea de las aportaciones de Bunbury (2020) o Schreiber (2017), es importante destacar la importancia que tiene diseñar las asignaturas desde el inicio siguiendo los principios del DUA, ya que esto evitaría tener que realizar ajustes sobre una asignatura ya planificada. A este respecto, como hace constar el análisis de los resultados de este estudio, del total del profesorado participante, solo cuatro ha planificado su asignatura teniendo en consideración la diversidad existente en las aulas desde el inicio, sin tener que hacer cambios sustanciales durante el desarrollo de su práctica docente. Esto puede deberse a la reciente aparición de esta línea de investigación y a los escasos estudios centrados en la aplicación de DUA en Educación Superior (Boothe et al., 2018; Fleet y Kondrashov, 2019). Con ello, desde este estudio se recomienda a las universidades, la creación de sólidos planes de formación continua para el profesorado, que aborden cuestiones relacionadas con el Diseño Universal de Aprendizaje y su aplicación en el aula.

\section{3. ¿Qué dificultades o necesidades encuentra el profesorado para realizar modificaciones en sus asignaturas?}

Respecto al tercer objetivo de este trabajo, se hace necesario abordar las dificultades que ha encontrado el profesorado para poder ajustar su enseñanza a las necesidades del alumnado con discapacidad, ya que merecen ser consideradas si se pretende avanzar hacia una Pedagogía Inclusiva en la universidad. Entre estas dificultades, el propio profesorado destaca su falta de formación especializada en atención al alumnado con discapacidad, hallazgo que coincide con las aportaciones de Moriña y Carballo (2018). Es por ello, que en este estudio se recomienda que se forme al profesorado sobre cómo enseñar y apoyar a los estudiantes con discapacidades, ya que esto mejoraría la calidad de la enseñanza y el aprendizaje de estos estudiantes.

El profesorado ha verbalizado que, en ocasiones, la información que reciben desde las Unidades de Apoyo al alumnado con discapacidad (encargadas de indicar los apoyos que necesita el alumnado con discapacidad, siempre que el alumnado comunique su condición y las adaptaciones que necesita) llega durante el desarrollo de las distintas asignaturas, lo cual dificulta que los apoyos se puedan 
ofrecer en tiempo y forma. Una posible solución para cubrir esta necesidad sería la contratación de más personal especializado en estas oficinas para agilizar los trámites conducentes a cubrir las necesidades del alumnado con discapacidad, la creación de sub-oficinas en cada una de las facultades, la mejora de los canales de comunicación para informar al profesorado sobre las características y necesidades del alumnado con discapacidad o la inclusión de planes de formación continua que contemplen la atención a la diversidad o la formación en materia de discapacidad (Autor, 2021).

A modo de síntesis, el estudio aporta algunas claves prácticas para seguir avanzando hacia la inclusión del alumnado con discapacidad en la universidad. A pesar de que el profesorado muestra actitudes positivas hacia la realización de modificaciones en sus asignaturas, admite tener ciertas dificultades para realizarlas, como la falta de formación en esta línea, o la falta de información sobre las necesidades de su alumnado al inicio de curso. Por su parte, entre las acciones realizadas, las adaptaciones de acceso a la comunicación son las más demandadas por el alumnado con discapacidad, aspecto tranquilizador, ya que indica que los ajustes que precisa el alumnado son sencillos en su mayoría, sin requerir grandes esfuerzos por parte del profesorado, ni grandes cambios en los proyectos docentes de las asignaturas. Con todo esto, se recomienda al personal encargado de tomar decisiones en las distintas universidades, la creación, por un lado, de planes de formación sólidos, centrados en Atención a la Diversidad del alumnado universitario y en el Diseño Universal de Aprendizaje, entre otras temáticas relacionadas; y por otro, la contratación de más personal técnico en los Servicios de Apoyo al alumnado con Discapacidad, o la creación de unidades de apoyo en las distintas facultades, para garantizar que el soporte que se proporciona tanto al alumnado con discapacidad como al profesorado, es adecuado y se ofrece con suficiente antelación para poder ajustar la enseñanza.

\section{Referencias bibliográficas}

ABDELLA, A. S. (2018). "Instructors' Willingness to Provide Instructional Accommodations for Students with Disabilities in Selected Universities of Ethiopia". International Journal of Inclusive Education, 22, 671-682. https://doi.org/10.1080/136031 16.2017.1396501

ALCANTUD, F., ÁVILA, V., y AsENSI, M, C. (2000). Integración de estudiantes con discapacidad en los estudios superiores. Valencia: Universitat de València. Recuperado de: http://sapdu.unizar.es/sites/default/files/108610_A4B3DF5Cd01.pdf 
BanKS, J. (2019). "Are We Ready: Faculty Perceptions of Postsecondary Students with Learning Disabilities at a Historically Black University". Journal of Diversity in Higher Education, 12(4), 297-306. https://doi.org/10.1037/dhe0000100

Boothe, K. A., LohmanN, M. J., Donnell, K. A., \& Hall, D. D. (2018). "Applying the Principles of Universal Design for Learning (UDL) in the College Classroom". The Journal of Special Education Aprenticeship, 7(3), 1-13.

BunBury, S. (2020). "Disability in higher education - do reasonable adjustments contribute to an inclusive curriculum?". International Journal of Inclusive Education, 24(9), 964-979. https://doi.org/10.1080/136031 16.2018.1503347

CAST (2018). Universal design for learning guidelines. Version 2.2. Wakefield, MA. Recuperado de: http://udlguidelines.cast.org/

Dalton, E. M., (2017). "Beyond Universal Design for Learning: Guiding Principles to Reduce Barriers to Digital \& Media Literacy Competence". Journal of Media LiteracyEducation, 9(2), 17-29. https://doi.org/10.23860/JMLE-2019-09-02-02

Debrand, C. C., \& Salzberg, C. L. (2005). "A Validated Curriculum to Provide Training to Faculty regarding Students with Disabilities in Higher Education". Journal of Postsecondary Education and Disability, 18(1), 49-61. Recuperado de: https:// files.eric.ed.gov/fulltext/EJ846380.pdf

Defez, A. (2005). "¿Qué es una creencia?". Logos. Anales Del Seminario De Metafísica, 38, 199-221. Recuperado de: https://revistas.ucm.es/index.php/ASEM/ article/view/ASEM0505110199A/15904

Elbeherl, G., Everatt, J., Theofanides, F., Mahfoudhi, A., \& Al Muhareb, K. (2018). "Attitudes of academics to special needs accommodations in Kuwait". International Journal of Inclusive Education, 1-15. https://doi.org/10.1080/13603116.201 8.1508517

Fichten, C. S., Heiman, T., Havel, A., Jorgensen, M., Budd, J., \& King, L. (2016). "Sustainability of Disability-Related Services in Canada and Israel: Will the Real Universal Design Please Stand Up?". Exceptionality Education International, 26(1), 19-35. https://doi.org/10.5206/eei.v26i1.7733

Fleet, C., \& Kondrashov, O. (2019). "Universal Design on University Campuses: A Literature Review". Exceptionality Education International, 29(1), 136-148. Recuperado de: https://ojs.lib.uwo.ca/index.php/eei/article/view/11125/8803 
FLORIAN, L. (2014). "What counts as evidence of inclusive education?" European Journal of Special Needs Education, 29(3), 286-295. doi:10.1080/08856257. 2014.933551

FUNDACIÓN UNIIERSIA (2018). "Universidad y Discapacidad. IV Estudio sobre el grado de inclusión del sistema universitario español respecto de la realidad de la discapacidad". https://www.cermi.es/sites/default/files/docs/colecciones/ Fundacion_IVEstudio_AAFF.pdf

Garín, J., Muñoz, J. L, Galán-Mañas, A., Fernández, M., y Sanahuja, J. (2013). "El plan de acción tutorial para estudiantes universitarios con discapacidad". Revista nacional e internacional de educación inclusiva, 6(3), 89-108. Recuperado de: https://ddd.vab.cat/pub/artpub/2013/147497/revnacint_a2013v6n3p89.pdf

Gale, T., \& Mills, C. (2013). "Creating Spaces in Higher Education for Marginalised Australians: Principles for Socially Inclusive Pedagogies". Enhancing Learning in the Social Sciences, 5(2), 7-19. https://doi.org/10.11120/elss.2013.00008

GiBSON, S. (2015). "When Rights Are Not Enough: What is? Moving towards New Pedagogy for Inclusive Education within UK Universities". International Journal of Inclusive Education, 19(8), 875-886. http://dx.doi.org/10.1080/13603116.2 015.1015177

Greenberger, L. (2016). "Faculty Attitudes toward Students with Disabilities at an Israeli Leading Technology Institute". Learning Disabilities: A Multidisciplinary Journal, 21(1), 1-13. https://doi.org/10.18666/LDMJ-2016-V21-I1-6814

HONG, B. S. (2015). "Qualitative analysis of the barriers college students with disabilities experience in higher education". Journal of College Student Development, 56(3), 209-226. http://dx.doi.org/10.1353/csd.2015.0032

Hromalik, C. D., Myhill, W. N., \& CarR, N. R. (2020). "All Faculty Should Take This": A Universal Design for Learning Training for Community College Faculty". TechTrends: Linking Research and Practice to Improve Learning, 64(1), 91-104. https://doi.org/10.1007/s11528-019-00439-6

Hsiao, F., Burgstahler, S., Johnson, T., Nuss, D., \& Doherty, M. (2019). "Promoting an Accessible Learning Environment for Students with Disabilities via Faculty Development (Practice Brief)". Journal of Postsecondary Education and Disability, 32(1), 91-99. Recuperado de: https://files.eric.ed.gov/fulltext/EJ1217448.pdf 
Kı̈O, V., \& MAKOelle, T. (2014). "Inclusion in Higher Education: Learning Experiences of Disabled Students at Winchester University". International Education Studies, 7(6), 106-116. http://dx.doi.org/10.5539/ies.v7n6p106

LANGØrGen, E., \& MAGNUS, E. (2018). "We are just ordinary people working hard to reach our goals! Disabled students' participation in Norwegian higher education". Disability \& Society, 33(4), 598-617. https://doi.org/10.1080/0968759 9.2018 .1436041

Ley Orgánica 4/2007, de 12 de abril, de universidades. Boletín Oficial del Estado, núm. 89, de 13 de abril de 2007, pp. 16241 a 16260. Recuperado de: https:// www.boe.es/buscar/doc.php?id=BOE-A-2007-7786

Lightfoot, A., Janeml, R., \& Rudman, D. L. (2018). "Perspectives of North American Postsecondary Students with Learning Disabilities: A Scoping Review". Journal of Postsecondary Education and Disability, 31(1), 57-74. Recuperado de: https:// files.eric.ed.gov/fulltext/EJ1 182368.pdf

Lombard, A., MurRay, C., \& Dallas, B. (2013). "University Faculty Attitudes toward Disability and Inclusive Instruction: Comparing Two Institutions". Journal of Postsecondary Education and Disability, 26(3), 221-232. Recuperado de: https://files. eric.ed.gov/fulltext/EJ1026882.pdf

Melero, N., Moriña, A., y Perera, V. H. (2019). "Acciones del profesorado para una práctica inclusiva en la universidad". Revista Brasileira de Educaçao, 24, e240016. http://dx.doi.org/10.1590/s1413-24782019240016

MeYER, A., Rose, D. H., \& GoRDon, D. (2014). Universal Design for Learning: Theory \& Practice. CAST Professional Publishing.

Miles, M. B., \& HuBerman, A. M. (1994). Qualitative Data Analysis: An Expanded Sourcebook (2nd Ed). Sage Publications.

MoriñA, A. (2020). "Approaches to Inclusive Pedagogy: A Systematic Literature Review". Pedagogika, 140(4), 134-154. https://doi .org/10.15823/p.2020.140

Moriña, A., Cortés-Vega, M. D., \& Molina, V. M. (2015). "Faculty training: An Unavoidable Requirement for Approaching More Inclusive University Classrooms". Teaching in Higher Education, 20(8), 795-806. https://doi.org/10.1080/1356 2517.2015.1085855 
Moriña, A. y Carballo, R. (2018). "Profesorado Universitario y educación inclusiva: respondiendo a sus necesidades de formación". Psicologia Escolar e Educacional, 22(Núm. Especial), 87-95. http://dx.doi.org/10.1590/2175-35392018053

Moriña, A., Perera, V. H., \& Melero, A. (2020). "Difficulties and reasonable adjustments carried out by Spanish faculty members to include students with disabilities". British Journal of Special Education, 47(1), 6-23. https://doi. org/10.1111/1467-8578.12261

MBuvHA, T. (2019) "Kinds of Support Offered by the Disability Unit to Students with Disabilities at Institutions of Higher Learning in South Africa: A Case Study of the University of Venda". Journal of Student Affairs in Africa, 7(2), 57-73. https:// doi.org/10.24085/jsaa.v7i2.3825

Real Decreto 1791/2010, de 30 de diciembre, por el que se aprueba el Estatuto del Estudiante Universitario. Boletín Oficial del Estado, núm. 318, de 31 de diciembre de 2010, pp.109353 a 109380. Recuperado de: https://www.boe.es/ eli/es/rd/2010/12/30/1791

Real Decreto Legislativo 1/2013, de 29 de noviembre, por el que se aprueba el Texto Refundido de la Ley General de derechos de las personas con discapacidad y de su inclusión social. Boletín Oficial del Estado, núm. 289, de 3 de diciembre de 2013, pp. 95635 a 95673 . Recuperado de: https://www.boe.es/eli/es/ $\mathrm{rdlg} / 2013 / 11 / 29 / 1 / \mathrm{dof} / \mathrm{spa} / \mathrm{pdf}$

Rodesiler, C., \& McGuire, J. (2015). "Ideas in Practice: Professional Development to Promote Universal Design for Instruction". Journal of Developmental Education, 38(2), 24-31. Recuperado de: http://www.jstor.org/stable/24614043

Rouse, M. (2009). "Developing inclusive practice: a role for teachers and teacher education". Education in the North, 16, 6-13.

Sánchez Díaz, M. N. \& Morgado, B. (en prensa). "Moving toward the inclusion of university students with disabilities. Barriers, facilitators and recommendations identified by inclusive faculty". The Journal of Continuing Higher Education.

Sandoval, M., Morgado, B., \& DOmÉneCH, A. (2020). "University students with disabilities in Spain: faculty beliefs, practices and support in providing reasonable adjustments", Disability \& Society. https://doi.org/10.1080/09687599.2020.1 751078 
SAPDU (2017). Guía de Adaptaciones en la Universidad. Recuperado de: http:// sapdu.unizar.es/sites/default/files/Guia\%20de\%20adaptaciones_DIGITAL.pdf

SCHREIBER, J. (2017). "Universal Design for Learning: A Student-Centered Curriculum Perspective". Curriculum and Teaching, 32(2), 89-98. https://doi.org/10.7459/ $\mathrm{ct} / 32.2 .06$

SCHREUER, N., \& SACHS, D. (2014). "Efficacy of accommodations for students with disabilities in higher Education. Journal of Vocational Rehabilitation, 40(1), 27-40. doi:10.3233/JVR-130665

Seale, J., Colwell, C., Coughlan, T., Heiman, T., Kaspi-Tsahor, D., \& Olenik-Shemesh, D. (2020). "'Dreaming in colour': disabled higher Education students' perspectives on improving design practices that would enable them to benefit from their use of technologies. Education and Information Technologies". https://doi.org/10.1007/ s10639-020-10329-7

ToutAIN, C. (2019). "Barriers to Accommodations for Students with Disabilities in Higher Education: A Literature Review". Journal of Postsecondary Education and Disability, 32(3), 297-310. Recuperado de: https://files.eric.ed.gov/fulltext/ EJ1236832.pdf 